\title{
Re: Neurology Research in Saudi Arabia Urgent call for action
}

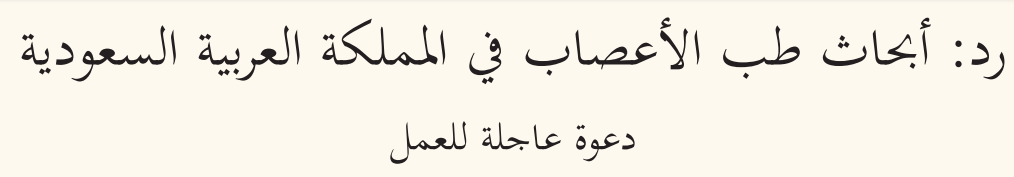

Sir,

I read with great interest the study by Algahtani et al. published in the August 2017 issue of SQUMJ in which a bibliometric analysis was performed of neurology-related articles published from Saudi Arabia over the past two decades. ${ }^{1}$ The findings from this study indicated substantial growth in the number of neurology-related publications from this country, particularly over the last four years. ${ }^{1}$ This is an encouraging trend that may perhaps reflect a high degree of research activity conducted in Saudi Arabia.

The quality and quantity of research produced in the Arabian Gulf has recently been the subject of scholarly attention. ${ }^{2,3}$ Similar to the findings reported in Algahtani et al's study, rising trends in biomedical research productivity have been reported in Saudi Arabia and other Arabian Gulf countries. ${ }^{1-4}$ However, such trends deserve further discussion. Algahtani et al. examined the quality of Saudi-based neurology publications using measures of research design and journal impact factor (IF); firstly, they found that the observed growth in neurology-related publications was mainly accounted for by a growing body of case reports/observational studies and, secondly, that more than half of the identified articles (55\%) were published in journals with low IFs. ${ }^{1}$

The validity of using the IF as a measure of quality in scholarly research has been extensively debated. ${ }^{5}$ While publication in prestigious high-impact journals may reflect high-quality research, it does not necessarily represent the value of that research within a local context. Furthermore, as most biomedical journals with high IFs are published in high-income Western countries (i.e. North America and Europe), they often focus on topics and health problems of interest to that region of the world. For these reasons, researchers working on projects of interest to the Arabian Gulf region often face challenges publishing their findings in top-tier international journals. ${ }^{6}$ In addition, some researchers may deem publication in local journals more appropriate than in highly-ranked international journals. In light of this, using a journal's IF as the sole indicator of research quality-especially for studies conducted in low-income countries and those with emerging economies, such as those in the Arabian Gulf region—-should be avoided.

Another proxy measure of research quality is the prevalence of scientific collaboration with other local (i.e. Gulf-based) and international research institutions. ${ }^{4}$ Collaboration between different research bodies within a country and/or with external institutions may reflect the complexity of the research and mirror an increasing trend in multicentre studies. Overall, in order to better examine the quality of neurology literature produced in Saudi Arabia, further research is required to evaluate patterns in authorship (i.e. the number of authors per publication over time) and local/international collaboration.

\section{Ibrahim S. Al-Busaidi}

Department of General Medicine, Christchurch Hospital, Canterbury District Health Board, Christchurch, New Zealand; Edgar Diabetes E Obesity Research Centre, Dunedin School of Medicine, University of Otago, Dunedin, New Zealand

E-mail:Ibrahim.Al-busaidi@cdhb.health.nz

\section{References}

1. Algahtani H, Shirah B, Boker F, Algamdi A, Alkahtani A. Neurology research in Saudi Arabia: Urgent call for action. Sultan Qaboos Univ Med J 2017; 17:e324-8. doi: 10.18295/squmj.2017.17.03.011.

2. Al-Bishri J. Evaluation of biomedical research in Saudi Arabia. Saudi Med J 2013; 34:954-9.

3. Al-Maawali A, Al Busadi A, Al-Adawi S. Biomedical publications profile and trends in Gulf Cooperation Council countries. Sultan Qaboos Univ Med J 2012; 12:41-7. doi: 10.12816/0003086. 
4. Moed HF. Iran's scientific dominance and the emergence of South-East Asian countries as scientific collaborators in the Persian Gulf region. Scientometrics 2016; 108:305-14. doi: 10.1007/s11192-016-1946-x.

5. Neuberger J, Counsell C. Impact factors: Uses and abuses. Eur J Gastroenterol Hepatol 2002; 14:209-11. doi: 10.1097/00042737-20020300000001.

6. Habibzadeh F. Scientific research in the Middle East. Lancet 2014; 383:e1-2. 